\title{
Clinical Outcomes of COVID-19 Cases and Influencing Factors of Severe Cases in Qingdao City: A Retrospective Cohort Study
}

Jing Jia

Qingdao Municipal Center for Disease Control and Prevention

Xiaoqi Dai

Qingdao Municipal Center for Disease Control and Prevention

Xiaolin Jiang

Shandong CDC: Shandong Center for Disease Control and Prevention

Shuo Li

The Affiliated Hospital of Qingdao University

\section{Xia Wang}

Qingdao Shibei Center for Disease Control and Prevention

\section{Ruqin Gao}

Qingdao Municipal Center for Disease Control and Prevention

Fachun Jiang

Qingdao Municipal Center for Disease Control and Prevention

Fei Qi

Qingdao Municipal Center for Disease Control and Prevention

Dianmin Kang ( 404870862@qq.com )

Shandong Center for Disease Control and Prevention

\section{Research article}

Keywords: COVID-19, Clinical outcomes, severe cases, influencing factor

Posted Date: October 26th, 2020

DOI: https://doi.org/10.21203/rs.3.rs-95094/v1

License: (c) (1) This work is licensed under a Creative Commons Attribution 4.0 International License.

Read Full License 


\section{Abstract}

\section{Background}

To analyze the clinical outcomes of COVID-2019 cases and the influencing factors of severe cases in Qingdao City and provide theoretical reference basis for optimizing medical treatment and the strategies of epidemic prevention and control.

\section{Methods}

The demographical, epidemiological, clinical data of 81 confirmed COVID-2019 cases in Qingdao City were collected via epidemiological investigation and clinical process tracking. The status of cure, discharge, clinical outcome and influencing factors were analyzed in our study.

\section{Results}

Among the 81 cases $\nabla 12(14.81 \%)$ and $55(67.90 \%)$ were mild and ordinary, $9(11.11 \%)$ and $4(4.94 \%)$ were severe and critical, and 1 critical cases (1.23\%) developed into fatal, with the fatality rate $1.23 \%$. The median time from onset of symptoms to hospital admission were 3.67 days (IQR, 1.75 to 6.71). The median duration of illness were 21.00 days (IQR, 16.00 to 26.00 ) and the median length of hospitalization were 15.63 days (IQR, 11.60 to 20.50). The median time for progression to severe cases was 6.00 days after onset (IQR, 5.00-10.00). The median duration of severe cases was 8.00 days (IQR, 6.25-14.00). Age older than 40 years old $(\mathrm{OR}=5.797,95 \% \mathrm{Cl}$ : 1.064 31.568) and first chest CT abnormal (OR=0.1140, 95\% Cl: 0.014 0.923) were the influencing factors of COVID-2019 severe cases.

\section{Conclusions}

Older age and first chest CT normal would be more prone to develop to severe cases of COVID-2019. During the epidemic period, it was necessary to classify and manage cases according to the needs of prevention and control in order to ensure the rational allocation of medical resources.

\section{Introduction}

Coronavirus disease 2019 (COVID-19), named by WHO (World Health Organization) on Feb 11, 2020, caused serious respiratory illness such as pneumonia and lung failure [1]. On March 11, 2020, WHO declared that COVID-19 was a pandemic [2]. COVID-19 was a human infectious disease caused by a recently emerged novel coronavirus (severe acute respiratory syndrome coronavirus 2 [SARS-CoV-2])[3, 4]. The typical symptoms were fever, sore throat, fatigue, cough or dyspnea, and so on[5-7]. The transmission of COVID-19 is potent. As of September 27, 2020, the outbreak of COVID-19 has spread to 235 countries, areas or territories, generated 32,730,945 confirmed cases, including 991,224 deaths worldwide, with rapid daily increases in some countries. 
Since December 2019, some cases had been linked to Huanan market, a seafood and wild animal market, suggesting animal-to-human transmission[8, 9]. At the end of 2019, the WHO was informed about an outbreak of pneumonia of unknown etiology in Wuhan, Hubei Province, China. Current evidence indicated that SARS-CoV-2 spread by human-to-human. It was mainly transmitted by symptomatic cases[10-12]. China had used two strategies, containment and suppression, to promote prevention and control[13]. The strategies effort has reduced morbidity and case-fatality ratio of COVID-19. As of September 27, 2020, a total of 85,372 cases of the COVID-19 were reported in 31 provinces (districts and cities) and Xinjiang Production and Construction Corps in China. Especially recently, the number of confirmed cases nationwide was few, and most of them were imported cases.

On 21 January 2020, the first COVID-19 case of Qingdao was confirmed. Although we control COVID-19 transmission effectively in the domestic, we are facing additional waves from imported cases. To be able to prevent future outbreaks, we studied the factors influencing the outcome of COVID-19 confirmed cases, based on the previous epidemic situation in Qingdao, Shandong Province, China.

\section{Materials And Methods}

\subsection{Data Collection}

The data of COVID-19 confirmed cases was obtained from the National Notifiable Disease Surveillance System. The demographics and epidemiological information (time of onset, admission, hospitalization; History of underlying diseases; clinical outcome, and so on) was included in the investigation report. The epidemiological questionnaire was collected onto standardized forms through interviews of cases. All epidemiologic information was collected during field investigations.

\subsection{Case and related definitions}

The criteria was as follows. A confirmed case was defined by positive respiratory specimens and clinical symptoms. The respiratory specimens was tested by real-time reverse-transcription-polymerase-chainreaction (RT-PCR) assay for SARS-CoV-2 or a genetic sequence that matches SARS-CoV-2. Clinical classification was based on the most serious clinical manifestations, which was divided into mild, ordinary, severe and critical. Among this clinical classification, mild and ordinary were defined as mild cases; severe, critical and fetal were defined as severe cases.

The duration of illness was from onset of symptoms to hospital discharge. The length of hospitalization was from hospital admission to discharge. The cure rate was defined as the probability of being cured from onset of symptoms to hospital discharge on the $n$ days of onset. The hospital discharge rate was defined as the probability of being cured from hospital admission to discharge on the $\mathrm{n}$ days of onset.

\subsection{Influencing factors assignment rule}

The assignment rule was as follows. Gender: Gender was controlled by women. Age: 0 to 39 years old was assigned as 1, above 40 years old was assigned as 2 . Occupation: Retired and unemployment was 
assigned as 1, students and children was assigned as 2, in-service staff and the others were assigned as 3 and 4. Time interval between onset and hospital admission: On the day from onset to hospital admission was assigned as $0.1-2 d, 3-4 d$, and more than $5 d$ from onset to hospital admission was assigned as 1, 2, 3, respectively. Imported cases from abroad, cluster cases, chest computed tomography (CT), body temperature, and close contacts were dichotomous variables.

\subsection{Laboratory Testing}

Upper or lower respiratory tract specimens were sampled from patients. The samples were divided and tested in biosafety level 2 facilities. Real-time RT-PCR with SARS-CoV-2 -specific primers and probes was used to test RNA which was extracted from the samples. The laboratory-confirmed case was diagnosed based on two targets (open reading frame $1 \mathrm{a}$ or $1 \mathrm{~b}$, nucleocap-sid protein) positive test by specific realtime RT-PCR. If a cycle threshold value (Ct-value) was less than 37, the result was positive, and a Ct-value of 40 or more was defined as negative. If the Ct-value was 37 to less than 40 , retested was required. A repeated Ct-value, tested less than 40 and observed an obvious peak, was defined as a positive result.

\subsection{Statistical analysis}

We carried out descriptive analysis for the categories. Continuous variables were reported as means with standard deviations or medians with ranges. The proportion was calculated for categorical variable. Pearson's chi-square test or Fisher's exact test was used to comparison analysis. Logistic regression analysis was used to analyze the influencing factors of severe cases. A P-value $<0.05$ was considered to indicate statistical significance. All analysis was performed with SAS software (SAS version 9.4) for Windows.

\section{Results}

\subsection{Demographical|lepidemiologic and clinical characteristics}

As the first confirmed case of COVID-19 in Qingdao was reported on Jan 22, 2020, the cumulative number of confirmed cases gradually increased (Fig 1). A total of 81 confirmed cases had been reported in Qingdao City until to May 2, 2020. Among these cases, 80 cases had been discharged from hospitals and 1 had been deceased.

Of 81 confirmed cases, 38 were males and 43 were females, with an average male-to-female sex ratio 0.88:1. The median age of cases was 42 years (range, 1 to 90 ). $82.72 \%$ of the cases were under 60 years old. A total of $24.69 \%$ of the cases had one or more coexisting underlying medical conditions (20 cases, including 7 with hypertension, 4 with gallstones, 4 with diabetes, 3 with coronary heart disease and 2 with other diseases). The majority of cases were retired and unemployment, which accounted for $33.33 \%$, following by in-service staffs (24.68\%) and students and children $(23.46 \%)$.

The most common symptom at onset of illness were fever (72.84\%) and cough (30.86\%); less common symptoms were fatigue (14.81\%), sputum production(13.58\%), myalgia(13.58\%) and headache(12.35\%). 
On admission, the blood counts in 10 cases showed leucopentia (white blood cell count less than 4 x109/L), and 25 cases showed lymphopenia (lymphocyte percentage<20\%). 53 (65.43\%) cases had bilateral ground-glass opacity in first chest CT images.

\subsection{Status of cure, discharge and clinical outcome}

The median time from onset of symptoms to hospital admission were 3.67 days (IQR, 1.75 to 6.71 ). The median duration of illness were 21.00 days (IQR, 16.00 to 26.00) and the median length of hospitalization were 15.63 days (IQR, 11.60 to 20.50), mild and severe cases of which were showed in Table 2, respectively. The cure rate of mild cases within 20,30 , and 40 days were respectively $50.75 \%, 85.07 \%$, and $98.51 \%$, while $42.86 \%, 64.29 \%$ and $85.71 \%$ for severe cases. The difference of the cure rate between mild and severe cases was statistically significant by chi-square test $(P<0.05)$. The hospital discharge rate of mild cases within 20,30 , and 40 days were respectively $77.61 \%, 97.01 \%$ and $98.51 \%$, while $57.14 \%$, $85.71 \%$, and $100.00 \%$ for severe cases. The difference of the hospital discharge rate between mild and severe cases was statistically significant by chi-square test $(P<0.05)$. Fig. 2.

At admission, $12(14.81 \%)$ and 55(67.90\%) were mild and ordinary, $9(11.11 \%)$ and $4(4.94 \%)$ were severe and critical among the 81 cases, and 1 critical cases (1.23\%) developed into fatal. The median time for progression to severe cases was 6.00 days after onset (IQR, 5.00-10.00). The median duration of severe cases was 8.00 days (IQR, 6.25-14.00). Fig 3.

\subsection{Univariate analysis of the influencing factors}

Table 2 summarized the results of univariate analysis. We evaluated the effect of each factor on severe cases by chi-square test or Fisher's exact test. Age, occupation, first chest CT and body temperature were statistically significant.

\subsection{Multivariate analysis of the influencing factors}

Multivariate Logistic Regression was performed with the significant factors detected by univariate analysis. Table 3 showed that age older than 40 years old (OR=5.797, 95\% Cl: 1.064 31.568) and first chest $\mathrm{CT}$ abnormal (OR=0.1140, 95\% Cl: 0.014 0.923) were the influencing factors of COVID-19 severe cases, suggesting that older age and first chest CT normal would be more prone to develop to severe cases of COVID-19.

Sensitivity analysis was conducted to ensure the reliability of the results. A general liner model (GLM) was performed to analyze the association between four significant factors selected by univariate analysis and severe cases. The results were largely consistent, showing older age and first chest CT normal were positively correlated with severe cases (Table 4).

\section{Discussion}


This study retrospectively analyzed the demographical, epidemiological, clinical data in a cohort of COVID-19 cases in Qingdao City, Shandong Province. In this study, the median age of cases was 42 years, ranging from 1 to 90 years, with the majority (53.09\%) being female, which was different from other studies[14, 15]. These differences may be related to exposure person, $43.21 \%(35 / 81)$ of which were close contacts and most belonging to attendants in our study. Unlike the SARS with hyperpyrexia and dyspnea, many studies including our results, had showed that the most common clinical symptoms were fever, cough, fatigue, and myalgia, which was suggestive of common cold[16]. It had been reported that SARSCoV-2 appeared to replicate efficiently in the upper respiratory tract and might cause less abrupt onset of symptoms [17]. However, Kim et al had found that fever was absent in $75.00 \%$ of cases at the time of admission and they did not timely realize that they had been infected with SARS-CoV-2, which could result in the virus spreading inadvertently [18].

Compared to SARS and MERS, the fatality rate of COVID-19 was greatly lower than SARS and MERS (10.90\% and $34.40 \%$, respectively) [19-21]. The fatality rate of COVID-19 in Qingdao City was $1.23 \%$, which was lower than that in Hubei and Wuhan in the early phase [22]. The emergence of this situation might be associated with the rapid and effective response of epidemic prevention and control in Qingdao City. 'Four early' measures, namely early detection, early isolation, early diagnosis and early treatment, had vastly reduced the occurrence of cases and deaths.

Studies had found that data for COVID-19 available to date revealed a wide clinical spectrum of disease consisting of mild, ordinary, severe and critical disease. The severe rate was $17.28 \%$ in Qingdao City, appreciably lower than Guangdong Province (16.40\%). Wen et al found that the proportion of severe cases with older age and underlying diseases was much higher in Beijing [23]. Likewise, 78.57\%(11/14) were over 50 years old and $42.86 \%(6 / 14)$ had underlying disease of the 14 severe case in our study. Chen et al also showed that older adult males with chronic comorbidities was highly susceptible to SARS-CoV-2 as a result of the weaker immune functions of these patients[24].In addition, some studies suggested that a substantial leucopentia and lymphopenia might cause damage to T lymphocytes leading to exacerbation of cases[25, 26].

This study provided a comprehensive description of the three intervals associated with the clinical course in COVID-19 cases in Qingdao City. The average time of onset of symptoms to hospital admission were 3.67 days, which was slightly shorter than SARS (3.8 days)[19]. The average time of onset of symptoms to hospital discharge (the duration of illness, 21.00 days) was in line with other studies in MERS, but shorter than SARS (23 days)[20, 21]. Zhang et al showed that the duration of illness (23 days) and the length of hospitalization (20 days) in COVID-19 cases in Guangdong Province were all longer than our results[27].The discrepancies between these studies might attribute to the physical and clinical condition of individual.

Multivariate analysis revealed that older age and first chest CT abnormal were the influencing factors of COVID-19 severe cases. This result provided some references for early identification of severe risk and reduction of severe and fatality rate. Older age had been prone to develop to poor clinical outcome[28]. 
The severity of COVID-19 was positively correlated with age[29]. The reason may be that, as the age got older, T-cell decreased in numbers and functions of controlling viral replication and host inflammatory response which made more difficult for the host cell immunity to eradicate the invasive pathogen[30]. The first chest CT abnormal was the protective factors for severe cases. This might be because sufficient attention were paid by doctors and patients due to abnormal CT and more targeted treatment and nursing were given, which reduced the likelihood of developing severe disease. $\mathrm{YH} \mathrm{H}$ et al had pointed out that some patients presented normally in $\mathrm{CT}$ and the appearance of pulmonary infiltrates may be delayed and the absence of pulmonary changes on initial imaging did not mean that pneumonia will not develop[31]. Many studies had found that $79.4 \%$ patients were infiltrated bilaterally from chest $\mathrm{CT}$, which was similar with our results[32, 33].

Our findings further verified the results that older age and first chest CT normal would be more prone to develop to severe cases of COVID-19. Therefore, age and CT should be the focus of diagnosis and treatment of COVID-19 cases. In conclusion, the course and clinical severity of confirmed COVID-19 cases were under control in Qingdao City, which thanked to the timeliness and effectiveness of prevention, control and treatment measures. During the epidemic period, it was necessary to classify and manage cases according to the needs of prevention and control in order to ensure the rational allocation of medical resources.

There were several limitations of this study. First, our sample size is relatively small in comparison with other studies. There were 81 confirmed COVID-19 cases in Qingdao City during the study period. Second, only confirmed cases were analyzed and asymptomatic infected persons were not included in our study. Third, only the clinical outcome of the confirmed cases was analyzed. At present, each confirmed case had been admitted to hospital and isolated for treatment in Qingdao, regardless of clinical classification. Hence, conclusion extrapolation should be cautious.

\section{Conclusions}

Older age and first chest CT normal would be more prone to develop to severe cases of COVID-2019. During the epidemic period, it was necessary to classify and manage cases according to the needs of prevention and control in order to ensure the rational allocation of medical resources.

\section{Declarations}

\section{Funding}

This work was financially supported by The study on epidemiological characteristics and Transmission mechanism of COVID-19 in Shandong Province (grant number: 2020SFXGFY02-1).

\section{Availability of data and materials}


The datasets used and/or analyzed during the current study are available on request to the corresponding author.

\section{Ethics approval and consent to participate}

None.

\section{Consent for publication}

Yes.

\section{Authors' contributions}

Jing Jia, Xiaoqi Dai and Xiaolin Jiang are co-authors of this study. These authores contributed equally to this work.

Jing Jia: Conceptualization, Writing - original draft.

Xiaoqi Dai: Formal analysis, Writing - original draft.

Xiaolin Jiang: Investigation, Writing - original draft.

Shuo Li: Formal analysis, Writing - original draft.

Xia Wang: Investigation, Writing - original draft.

Ruqin Gao: Conceptualization, Writing - original draft.

Fachun Jiang: Conceptualization, Writing - original draft.

Fei Qi: Conceptualization, Writing - original draft.

Dianmin Kang: Conceptualization, Writing - original draft.

\section{Competing interest}

The authors declare that they have no known competing financial interests or personal relationships that could have appeared to influence the work reported in this paper.

\section{Acknowledgement}

We are deeply thankful to all health-care workers involved in the diagnosis and treatment of patients in Qingdao.

\section{References}


[1]. Zhu, N., et al., A Novel Coronavirus from Patients with Pneumonia in China, 2019. New England Journal of Medicine, 2020. 382: 727-33.

[2]. WHO, WHO characterizes COVID-19 as a pandemic. 2020, World Health Organization: Geneva. 2019.

[3]. Chu, J., et al., Clinical characteristics of 54 medical staff with COVID-19: A retrospective study in a single center in Wuhan, China. J Med Virol, 2020. 92: 807-13.

[4]. Yu, F., et al., Quantitative Detection and Viral Load Analysis of SARS-CoV-2 in Infected Patients. Clin Infect Dis, 2020.

[5]. Zhai, P., et al., The epidemiology, diagnosis and treatment of COVID-19. Int J Antimicrob Agents, 2020. 55: 105955.

[6]. Huang, X., et al., Epidemiology and Clinical Characteristics of COVID-19. Arch Iran Med, 2020. 23: 26871.

[7]. Fadini, G.P., et al., Prevalence and impact of diabetes among people infected with SARS-CoV-2. J Endocrinol Invest, 2020. 43: 867-69.

[8]. Chen, N., et al., Epidemiological and clinical characteristics of 99 cases of 2019 novel coronavirus pneumonia in Wuhan, China: a descriptive study. Lancet, 2020. 395: 507-13.

[9]. Chan, J.F., et al., A familial cluster of pneumonia associated with the 2019 novel coronavirus indicating person-to-person transmission: a study of a family cluster. Lancet, 2020. 395: 514-23.

[10]. Zhang, C., et al., Clinical and epidemiological characteristics of pediatric SARS-CoV-2 infections in China: A multicenter case series. PLoS Med, 2020. 17: e1003130.

[11]. Henry, B.M. and J. Vikse, Clinical Characteristics of Covid-19 in China. N Engl J Med, 2020. 382: 1860-61.

[12]. Zhu, N., et al., A Novel Coronavirus from Patients with Pneumonia in China, 2019. N Engl J Med, 2020. 382: 727-33.

[13]. Li, Z., et al., Active case finding with case management: the key to tackling the COVID-19 pandemic. Lancet, 2020. 396: 63-70.

[14]. Rothan, H.A. and S.N. Byrareddy, The epidemiology and pathogenesis of coronavirus disease (COVID19) outbreak. J Autoimmun, 2020. 109: 102433.

[15]. Adhikari, S.P., et al., Epidemiology, causes, clinical manifestation and diagnosis, prevention and control of coronavirus disease (COVID-19) during the early outbreak period: a scoping review. Infect Dis Poverty, 2020. 9: 29. 
[16]. Kuiken, T., et al., Newly discovered coronavirus as the primary cause of severe acute respiratory syndrome. Lancet, 2003. 362: 263-70.

[17]. Wu, V.C., et al., Acute renal failure in SARS patients: more than rhabdomyolysis. Nephrol Dial Transplant, 2004. 19: 3180-82.

[18]. Kim, E.S., et al., Clinical Course and Outcomes of Patients with Severe Acute Respiratory Syndrome Coronavirus 2 Infection: a Preliminary Report of the First 28 Patients from the Korean Cohort Study on COVID-19. J Korean Med Sci, 2020. 35: e142.

[19]. Feng, D., et al., Duration of symptom onset to hospital admission and admission to discharge or death in SARS in mainland China: a descriptive study. Trop Med Int Health, 2009. 14 Suppl 1: p. 28-35.

[20]. Oh, M.D., et al., Middle East respiratory syndrome: what we learned from the 2015 outbreak in the Republic of Korea. Korean J Intern Med, 2018. 33: 233-46.

[21]. Choi, W.S., et al., Clinical Presentation and Outcomes of Middle East Respiratory Syndrome in the Republic of Korea. Infect Chemother, 2016. 48: 118-26.

[22]. Huang, C., et al., Clinical features of patients infected with 2019 novel coronavirus in Wuhan, China. Lancet, 2020. 395: 497-506.

[23]. Ke, W., et al., Epidemiological and clinical characteristics of 46 newly-admitted coronavirus disease 2019 cases in Beijing.In Chinese. Chin J Infect Dis, 2020. 38.

[24]. Chen, N., et al., Epidemiological and clinical characteristics of 99 cases of 2019 novel coronavirus pneumonia in Wuhan, China: a descriptive study. Lancet, 2020. 395: 507-13.

[25]. Wong, C.K., et al., Plasma inflammatory cytokines and chemokines in severe acute respiratory syndrome. Clin Exp Immunol, 2004. 136: 95-103.

[26]. Chen, T.T., et al., TIM-2 is expressed on B cells and in liver and kidney and is a receptor for H-ferritin endocytosis. J Exp Med, 2005. 202: 955-65.

[27]. Yingtao, Z., et al., Clinical outcomes of COVID-19 cases and influencing factors in Guangdong province.In Chinese. Chin J Epidemiol, 2020. 41.

[28]. Wang, W., J. Tang and F. Wei, Updated understanding of the outbreak of 2019 novel coronavirus (2019-nCoV) in Wuhan, China. J Med Virol, 2020. 92: 441-47.

[29]. McMichael, T.M., et al., COVID-19 in a Long-Term Care Facility - King County, Washington, February 27-March 9, 2020. MMWR Morb Mortal Wkly Rep, 2020. 69: 339-42.

[30]. Smetana, J., et al., Influenza vaccination in the elderly. Hum Vaccin Immunother, 2018. 14: 540-49. 
[31]. Guo, Y.R., et al., The origin, transmission and clinical therapies on coronavirus disease 2019 (COVID19) outbreak - an update on the status. Mil Med Res, 2020. 7: 11.

[32]. Xu, B., et al., Epidemiological data from the COVID-19 outbreak, real-time case information. Sci Data, 2020. 7: 106.

[33]. Xu, X.W.W.X., Clinical findings in a group of patients infected with the 2019 novel coronavirus (SARSCov-2) outside of Wuhan, China: retrospective case series. BMJ (Clinical research ed.), 2020. m606.

\section{Tables}

Table 1 The duration of illness and hospitalization between mild and severe cases in Qingdao City

\begin{tabular}{|lllllll|}
\hline Type of Cases & \multicolumn{3}{c}{ duration of illness(days) } & \multicolumn{3}{c|}{ duration of hospitalization(days) } \\
\cline { 2 - 7 } & Median & $\mathrm{P}_{25}$ & $\mathrm{P}_{75}$ & Median & $\mathrm{P}_{25}$ & $\mathrm{P}_{75}$ \\
\hline Mild cases & 21.00 & 16.00 & 26.00 & 16.00 & 11.60 & 20.08 \\
\hline Severe cases & 24.5 & 16.50 & 33.75 & 17.00 & 12.23 & 23.54 \\
\hline
\end{tabular}

Table 2 Univariate analysis of the influencing factors of severe cases in Qingdao City $(n=81)$ 


\begin{tabular}{|c|c|c|c|c|c|}
\hline Characteristic & Mild Cases & $\begin{array}{l}\text { Severe } \\
\text { Cases }\end{array}$ & Total & $x^{2}$ & P-value \\
\hline Sex & & & & 0.1122 & 0.7376 \\
\hline Female & $35(81.40)$ & $8(18.60)$ & 43 & & \\
\hline Male & $32(84.21)$ & $6(15.79)$ & 38 & & \\
\hline Age & & & & 8.5464 & $0.0035^{\star}$ \\
\hline$\varangle 40$ & $37(94.87)$ & $2(5.13)$ & 39 & & \\
\hline$\geq 40$ & $30(71.43)$ & $12(28.57)$ & 42 & & \\
\hline Occupation & & & & 4.2076 & $0.0447 *$ \\
\hline Retired and unemployment & 19(70.37) & $8(29.63)$ & 27 & & \\
\hline Students and children & 19(100.00) & $0(0.00)$ & 19 & & \\
\hline in-service staff & $17(85.00)$ & $3(15.00)$ & 20 & & \\
\hline Other & $12(80.00)$ & $3(20.00)$ & 15 & & \\
\hline Underlying medical conditions & & & & 2.1457 & 0.0979 \\
\hline Yes & $14(70.00)$ & $6(30.00)$ & 20 & & \\
\hline No & $53(86.89)$ & $8(13.11)$ & 61 & & \\
\hline $\begin{array}{l}\text { Time interval between onset and hospital } \\
\text { admission }\end{array}$ & & & & 3.0167 & 0.0607 \\
\hline 0 & $1(100.00)$ & $0(0.00)$ & 1 & & \\
\hline 1 & $20(90.91)$ & $2(9.09)$ & 22 & & \\
\hline 2 & $24(92.31)$ & $2(7.69)$ & 26 & & \\
\hline 3 & $22(68.75)$ & $10(31.25)$ & 32 & & \\
\hline Imported cases from abroad & & & & 3.0012 & 0.0606 \\
\hline Yes & 16(100.00) & $0(0.00)$ & 16 & & \\
\hline No & $51(78.46)$ & $14(21.54)$ & 65 & & \\
\hline Cluster cases & & & & 2.1755 & 0.1402 \\
\hline Yes & $39(78.00)$ & $11(22.00)$ & 50 & & \\
\hline No & $28(90.32)$ & $3(9.68)$ & 31 & & \\
\hline First Chest CT & & & & 6.8982 & $0.0086^{*}$ \\
\hline Normal & $27(96.43)$ & $1(3.57)$ & 28 & & \\
\hline
\end{tabular}




\begin{tabular}{|c|c|c|c|c|c|}
\hline Abnormal & $40(75.47)$ & $13(24.53)$ & 53 & & \\
\hline Body Temperature & & & & 6.0245 & $0.0088^{*}$ \\
\hline Normal & $22(100.00)$ & $0(0.00)$ & 22 & & \\
\hline Abnormal & $45(76.27)$ & $14(23.73)$ & 59 & & \\
\hline Close Contacts & & & & 0.0009 & 0.9766 \\
\hline Yes & $29(82.86)$ & $6(17.14)$ & 35 & & \\
\hline No & $38(82.61)$ & $8(17.39)$ & 46 & & \\
\hline Total & $67(82.72)$ & $14(17.28)$ & 81 & & \\
\hline
\end{tabular}

Table 3 Multivariate analysis of the influencing factors of severe cases in Qingdao City $(n=81)$

\begin{tabular}{|c|c|c|c|c|c|c|c|}
\hline $\begin{array}{l}\text { influencing } \\
\text { factor }\end{array}$ & $\begin{array}{l}\text { Reference } \\
\text { group }\end{array}$ & $\beta$ & $\begin{array}{l}\text { Standard } \\
\text { Error }\end{array}$ & $\begin{array}{l}\text { Wald } \\
x^{2}\end{array}$ & $\begin{array}{l}\text { P- } \\
\text { Value }\end{array}$ & OR & $95 \% \mathrm{Cl}$ \\
\hline \multicolumn{8}{|l|}{ Age } \\
\hline$\geq 40$ & $₫ 40$ & 2.0015 & 0.8023 & 6.2233 & 0.0126 & 5.797 & $1.164 \sim 31.568$ \\
\hline \multicolumn{8}{|l|}{ Occupation } \\
\hline $\begin{array}{l}\text { Retired and } \\
\text { unemployment }\end{array}$ & other & -11.0796 & 196.8 & 0.0032 & 0.9551 & 1.387 & $0.0237 \sim 5.078$ \\
\hline $\begin{array}{l}\text { Students and } \\
\text { children }\end{array}$ & & -0.7636 & 1.0003 & 0.5827 & 0.4453 & 0.466 & $0.066 \sim 3.310$ \\
\hline In-service staff & & 0.2775 & 0.9290 & 0.0892 & 0.7652 & 1.320 & $0.214 \sim 8.151$ \\
\hline \multicolumn{8}{|l|}{ First Chest CT } \\
\hline Abnormal & Normal & -2.1717 & 1.0671 & 4.1416 & 0.0418 & 0.1140 & $0.014 \sim 0.923$ \\
\hline \multicolumn{8}{|l|}{$\begin{array}{l}\text { Body } \\
\text { Temperature }\end{array}$} \\
\hline Abnormal & Normal & -12.7486 & 209.8 & 0.0037 & 0.9516 & 1.3467 & $0.024 \sim 19.021$ \\
\hline
\end{tabular}

\section{Figures}




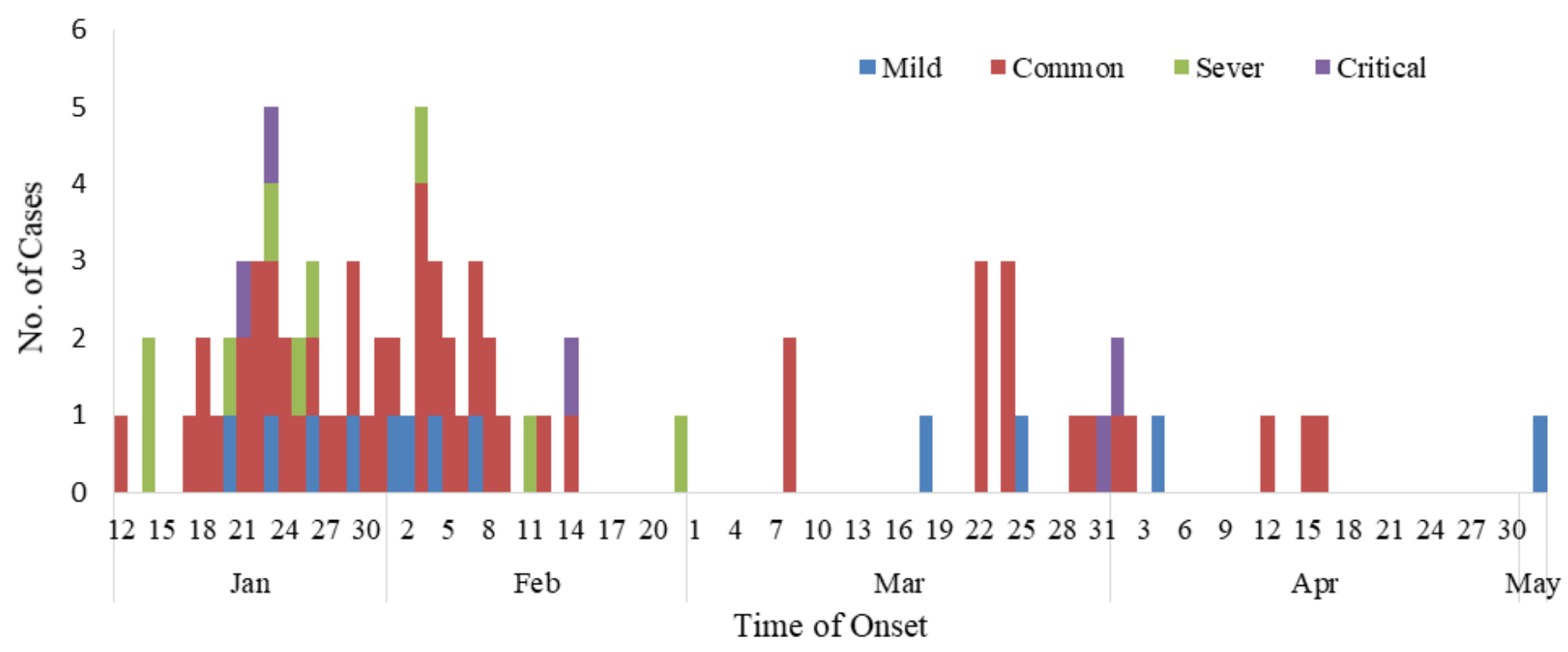

\section{Figure 1}

The time distribution of 81 COVID-19 confirmed cases in Qingdao from Jan 12 to May 2
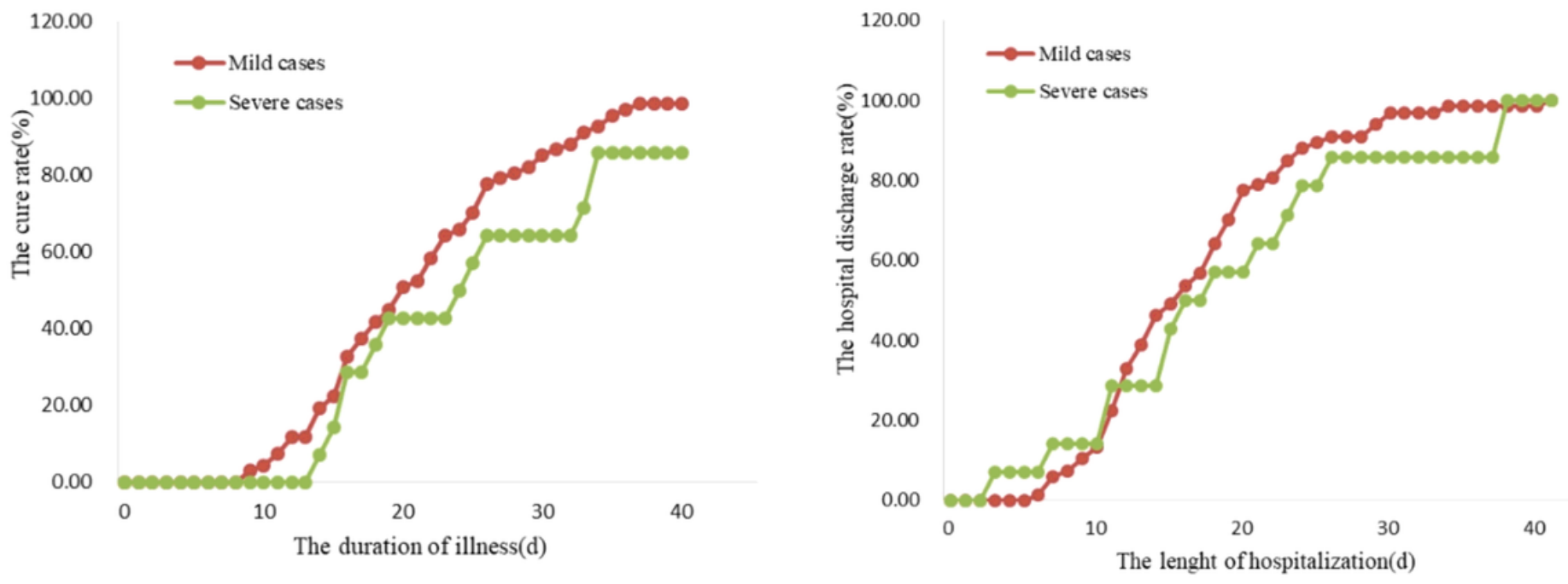

Figure 2

The cure rate and hospital discharge rete of mild and severe COVID-19 cases in Qingdao City 


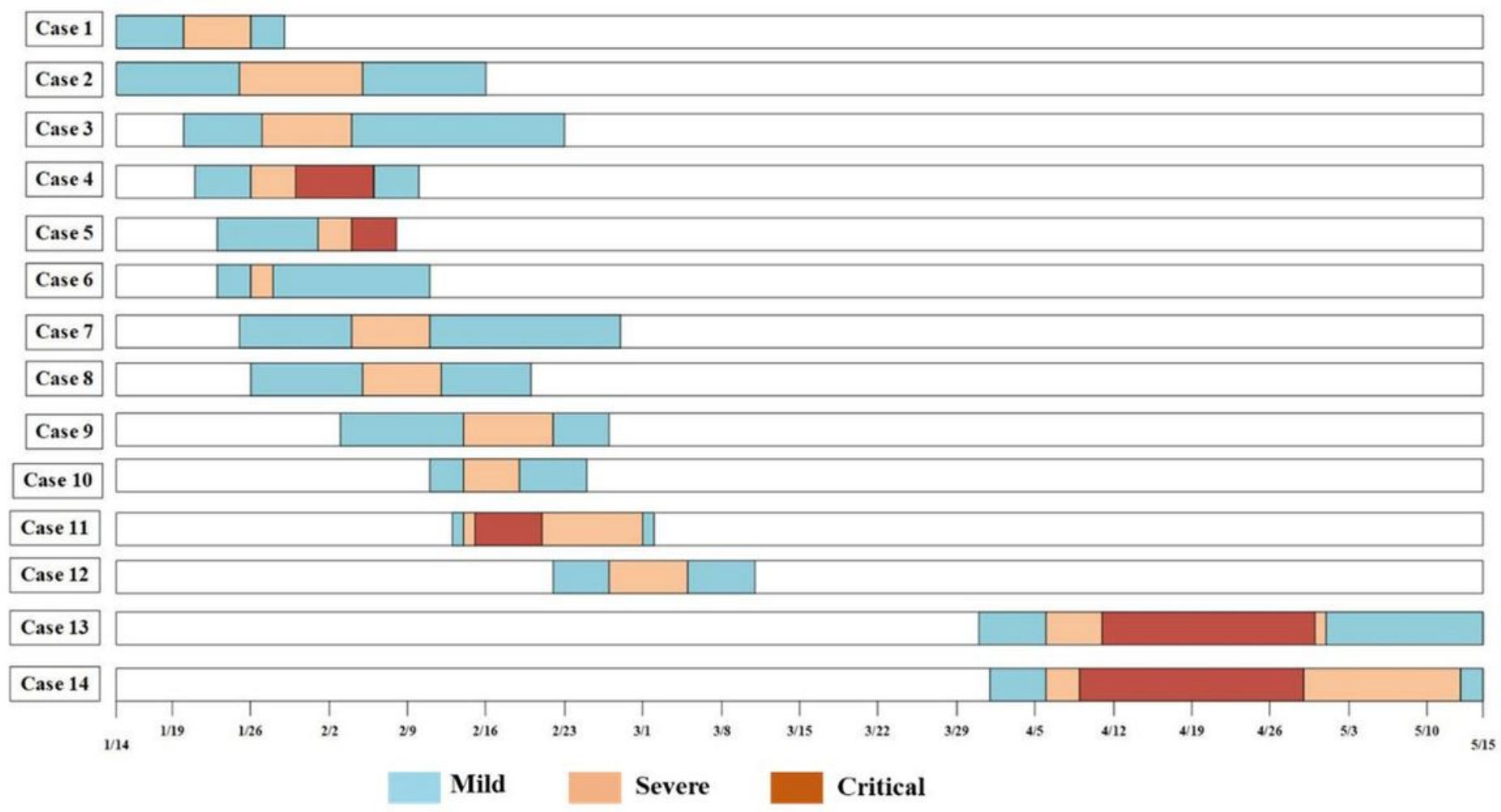

Figure 3

The clinical progress of severe COVID-19 cases in Qingdao City 